\title{
Office Hysteroscopic Treatment of Uterine Fibroids
}

\section{Ertan Saridogan, Christine S.Deguara}

University College London Hospitals, Women's Health Division, 250 Euston Road London NW1 2PG United Kingdom

\begin{abstract}
Advances in endoscopic and therapeutic hysteroscopic technology have made removal of submucosal fibroids possible in the outpatients setting. Removal of submucosal fibroids can be particularly challenging in the outpatients due to intramural components of some submucosal fibroids and the hard consistency of fibroids which makes specimen retrieval rather difficult through the endocervical canal. Fibroids which are $<2 \mathrm{~cm}$ and completely intracavitary are easier to remove in the outpatients. Specimen retrieval can be addressed either by slicing the fibroid using bipolar electrodes, by using a hysteroscopic morcellator, or leaving the fibroid in the uterine cavity after enucleation to be expelled with uterine contractions. Patient acceptability appears to be high in a small number of retrospective case series published in the literature. Further reports and data from prospective trials would be beneficial in improving our understanding of this procedure which appears to be performed by a relatively small number of centres.
\end{abstract}

Key words: Outpatient hysteroscopy, office hysteroscopy, submucosal fibroids, hysteroscopic myomectomy, fibroid resection, hysteroscopic morcellator

\section{Introduction}

Uterine fibroids are found in approximately $70-80 \%$ women before menopause and they develop in black women at an earlier age compared to white women.(1) Submucosal fibroids are more likely to be associated with menstrual disorders, recurrent miscarriages and infertility.(2) Despite their troublesome nature, submucosal fibroids are easier to treat surgically, due their accessibility via the hysteroscopic route. Hysteroscopy enables direct visualisation of the uterine cavity for the diagnosis and treatment of intrauterine pathology, including submucosal fibroids. Hysteroscopy is considered by many the gold standard for the diagnosis of submucous fibroids with the added benefit of offering the opportunity to see-and-treat concomitantly at the time of diagnosis.(3;4) Hysteroscopic 
myomectomy has been accepted as the standard treatment for submucous fibroids and is effective and safe in the management of menstrual disorders with the potential for better reproductive outcomes. (2)

The advances in endoscopic technology with higher image quality, development of smaller hysteroscopes with operative channels and ancillary instrumentation have facilitated operative hysteroscopic procedures to be performed in the outpatient or 'office' setting,(5) eliminating the morbidity associated with general anaesthesia. It is therefore described as a simple, safe, reproducible technique which is effective, quick, well-tolerated, and low-cost surgical procedure, with no need for an operating room.

The majority of operative outpatient hysteroscopies are for removal of polyps, but other procedures include removal of 'missing' intrauterine contraceptive devices, division of adhesions and removal of submucosal fibroids.(4) Removal of submucosal fibroids can be particularly challenging in the outpatients due to intramural components of some submucosal fibroids and the hard consistency of fibroids which makes specimen retrieval rather difficult through the endocervical canal. Over the years different groups described a number of strategies to overcome these difficulties for the removal of submucosal fibroids in the outpatient setting. The traditional method uses minihysteroscopes with an operating channel in conjunction with mechanical instruments and bipolar electrodes. More recently miniresectoscopes and hysteroscopic morcellators have been used in the outpatient setting to remove intrauterine fibroids.

\section{Technique}

\section{Hysteroscopic myomectomy using minihysteroscopes}

The conventional technique to remove submucosal fibroids in the outpatient setting involves slicing the fibroid into smaller pieces that would be possible to remove through the endocervical canal before detaching them from the uterine wall. This is then followed by retrieval of the slices using a hysteroscopic forceps.(6) Fibroids smaller then $0.5 \mathrm{~cm}$ would usually be possible to remove without slicing. Minihysteroscopes with an operating channel are used for this technique. Currently available minihysteroscopes allow use of 5-7 Fr instruments. $5 \mathrm{Fr}$ bipolar electrodes are usually used to slice the fibroids. An initial incision is made to divide the fibroid into two, starting from the free edge to the base. This is then followed by further incisions to slice the fibroid until the pieces will be small enough to pass through the cervix. The base of the fibroid is detached from its bed. The slices are 
then retrieved using hysteroscopic grasping forceps or biopsy cups which tend to work better due to better grasping capability.

For submucosal fibroids with an intramural component (Type I or II), it is suggested that the fibroid is first separated from its pseudocapsule at the base using mechanical instruments (hysteroscopic scissors and/or grasping forceps) until the intramural portion becomes intracavitary. This is followed by slicing the fibroid as described above.(6)

Using this method type 0 submucosal fibroids of up to $2 \mathrm{~cm}$ and type I or II fibroids of $<1.5 \mathrm{~cm}$ size may be possible to remove in the outpatient setting. $(4 ; 6)$ Preoperative administration of gonadotrophin releasing hormone analogues may facilitate the procedure by reducing the fibroid size. Very small fibroids $(0.5 \mathrm{~cm}$ or smaller) can usually be removed using mechanical instruments such as hysteroscopic scissors which are used to detach them from the fibroid bed, followed by retrieval with grasping forceps or biopsy cup without a need to slicing.

Some clinicians leave the fibroid in the uterine cavity after detaching it from the uterine wall, this is usually followed by liquefaction and expulsion of the fibroid with uterine contractions over the following few weeks.(7)

\section{Hysteroscopic myomectomy using miniresectoscope}

The traditional resectoscope with its $26 \mathrm{Fr}$ size requires cervical dilatation in most women and is difficult to use in the outpatient setting. Miniresectoscopes ( $16 \mathrm{Fr}$ or $5.3 \mathrm{~mm}$ ) which have been developed on the basis of paediatric resectoscopes have been used by some groups to resect submucosal fibroids in the outpatient setting.(8) This device allows procedures to be carried out with little or no cervical dilatation and is suitable for resection of relatively small fibroids using the traditional resection technique.

The traditional resectoscope has also been used for resection of fibroids in the outpatients.(9) This approach requires dilatation of the cervix to 8-10 $\mathrm{mm}$, depending on the resectoscope used, under local anaesthesia followed by resection of the fibroid using the traditional resection technique.

\section{Hysteroscopic morcellator or shaver}

Hysteroscopic morcellation uses specially designed $0^{\circ}$ hysteroscopes incorporating an offset proximal eyepiece to allow passage of a rigid, disposable, mechanical morcellating instrument down 
a straight working channel. The morcellator consists of a hollow tube which houses a rotating and/or reciprocating tubular hollow blade and is connected to an electrical generator and suction apparatus. Both tubes have a window opening at the end and the submucosal fibroid is sucked into this opening and 'shaved' by the rotating or oscilating blade. Cut pieces of the fibroid are simultaneously removed through the channel of the blade and captured at the suction device for histological analysis.(10) Avoidance of fibroid 'chips' may enhance intraoperative visualization and the simultaneous removal of fibroid tissue avoids the need for blind instrumentation to retrieve the specimen from the uterine cavity.

Recently a combined approach using a $5 \mathrm{Fr}$ bipolar needle electrode and morcellator was described to remove type I and II submucosal fibroids which have an intramural component.(11) A bipolar needle is used to circumscribe the leiomyoma and enter the pseudocapsule, thereby "releasing" the lesion from its bed. Following this a hysteroscopic morcellating system is then used to further dissect and remove the target lesion with electromechanical morcellation.

\section{Two step office myomectomy}

In this approach, two procedures are carried out with a four-week interval.(12) The first procedure involves the OPPluM (Office Preparation of Partially Intramural Myomas) technique which consists of an incision of the endometrial mucosa and pseudocapsule covering the myoma by means of hysteroscopic scissors, bipolar electrode or diode laser, along its reflection line on the uterine wall. The incision is deepened to the cleavage plane between the myoma and its pseudo-capsule.(13) This procedure promotes protrusion of the intramural portion of the myoma into the uterine cavity during the following menstrual cycles, thus facilitating the subsequent total removal of the lesion during the second procedure. The second procedure involves removal of the fibroid using diode laser or mechanical instrument such as scissors and grasping forceps four weeks later. Removal of type I or II submucosal fibroids of up to $4 \mathrm{~cm}$ was described with this approach, although, the myoma was left free in the uterine cavity when extraction was not possible due to the size of the mass, and an ultrasound check-up was performed 2 months later to assess the presence or absence of myoma remnants.(12) Although not reported in the literature, the second step can be carried out using the hysterosocpic morcellator.

The original OPPluM procedure was described prior to a traditional resection procedure under general anaesthesia and converts type I and II fibroids to completely or mostly intracavitary fibroids in more than $90 \%$ of the cases.(13) 


\section{Outcomes}

There are relatively few reports on the outcomes of outpatient hysteroscopic myomectomy. In our own experience $16 \%(23 / 142)$ of submucosal fibroids of all sizes was possible to remove in the outpatient setting and our cut-off for attempting to remove a fibroid was $2 \mathrm{~cm}$.(4)

Bettochi et al described their experience with removal of 49 type 0 fibroids of $\leq 2 \mathrm{~cm}$ and 21 Type I/II fibroids of $\leq 1.5 \mathrm{~cm}$. They stated that it was time consuming to attempt removing fibroids of $>2 \mathrm{~cm}$ in the office setting and that the results were suboptimal. Fibroids with an intramural portion (type I/II) had been treated with a three month course of GnRHa. These authors did not clarify whether there were any failures and the pain scores were none/mild (0-4/10) in $85 \%$ and $75 \%$ of patients with type 0 fibroids and type I/II fibroids, respectively. The remaining had moderate (5-7/10) pain scores and none reported severe pain.

Haimovich et al(12) reported on the otcome of their two step approach for office hysteroscopic resection of type I or II submucosal fibroids of $\leq 4 \mathrm{~cm}$. They were able remove all fibroids of $\leq 18 \mathrm{~mm}$ $(17 / 17)$ and $85 \%(17 / 20)$ of fibroids $19-30 \mathrm{~mm}$. None $(0 / 6)$ of fibroids $<30 \mathrm{~mm}$ was possible remove completely. In the nine patients in whom the fibroid was left free in the uterine cavity complete disappearance was confirmed by ultrasound two months after the second step, however it is unclear whether these patients had a third procedure. In the experience of this group, it was easier to enucleate fibroids on the anterior and posterior walls, compared to those with fundal or lateral wall location. These authors concluded that it was possible to remove submucosal fibroids $<3 \mathrm{~cm}$ and located on the anterior and posterior wall within the outpatient setting.

In the report by Haimovich et al(12) the VAS score for the first and second procedures were 2.5/10 and $3.0 / 10$ respectively. The satisfaction scores were $3.5 / 5$, with $75 \%$ of the patients reporting $3 / 5 \geq$ satisfaction rates.

These authors reported on further 61 patients with office hysteroscopic myomectomy, using a similar technique.(14) It is not clear whether the patients which were included in the previous report were included again in this second report, although there is overlap of the times of these two studies. The average size of the fibroid was $22 \mathrm{~mm}$ and the fibroid was left in the uterine cavity after enucleation. An ultrasound carried out 60-90 days after the procedure confirmed absence of fibroid in all cases. The patient satisfaction rates were very high (4.5/5). 
Papalampros et al(8) reported that four type ) or I submucosal fibroids of $<3 \mathrm{~cm}$ were successfully removed using the mini-resectoscope without using any local anaesthesia.

Currently, there are no outcome reports with the use of hysteroscopic morcellator for fibroid removal in the office setting.

\section{Pain relief for office hysteroscopic myomectomy}

The approaches to pain control for office hysteroscopic myomectomy varies in reports. Bettochi et al(6) do not use any anaesthesia or analgesia, whilst other groups may use local anaesthesia(11) or non-steroidal anti-inflammatory drugs with or without sedatives or opiates.(12;14)

\section{Conclusion}

Removal of submucosal fibroids in the office setting is possible and successful in selected patients. Fibroids which are $<2 \mathrm{~cm}$ and completely intracavitary are easier to remove in the outpatients. Removal of the intramural component of type I or II fibroids and specimen retrieval are the two main challenges of office hysteroscopic myomectomy. Specimen retrieval can be addressed either by slicing the fibroid using bipolar electrodes, by using a hysteroscopic morcellator, or leaving the fibroid in the uterine cavity to be expelled with uterine contractions. The strategies to remove fibroids with an intramural component include shrinkage with GnRHa, the OPPluM technique and a two step approach. It appears that the office hysteroscopic myomectomy offers all the benefits of outpatient procedures including avoidance of general anaesthesia and cost savings. Patient acceptability appears to be high in a small number of retrospective case series published in the literature. Data on the outcome of office hysteroscopic morcellator for myomectomy are likely to appear in the literature in the near future. Further reports and data from prospective trials would be beneficial in improving our understanding of this procedure which appears to be performed by a relatively small number of centres.

Reference List

(1) Baird DD, Dunson DB, Hill MC, Cousins D, Schectman JM. High cumulative incidence of uterine leiomyoma in black and white women: ultrasound evidence. Am J Obstet Gynecol 2003 Jan;188(1):100-7. 
(2) Saridogan E, Cutner A. Endoscopic management of uterine fibroids. Hum Fertil (Camb ) 2006 Dec;9(4):201-8.

(3) Clark TJ, Gupta JK. Handbook of outpatient hysteroscopy: a complete guide to diagnosis and therapy. London: Hodder Arnold; 2005.

(4) Gulumser C, Narvekar N, Pathak M, Palmer E, Parker S, Saridogan E. See-and-treat outpatient hysteroscopy: an analysis of 1109 examinations. Reprod Biomed Online 2010 Mar;20(3):423-9.

(5) RCOG, BSGE. Best practice in outpatient hysteroscopy. London: RCOG; 2011 Mar 1.

(6) Bettocchi S, Ceci O, Di VR, Pansini MV, Pellegrino A, Marello F, et al. Advanced operative office hysteroscopy without anaesthesia: analysis of 501 cases treated with a 5 Fr. bipolar electrode. Hum Reprod 2002 Sep;17(9):2435-8.

(7) Saridogan E. Surgical treatment of fibroids in heavy menstrual bleeding. Womens Health (Lond Engl ) 2016 Jan;12(1):53-62.

(8) Papalampros P, Gambadauro P, Papadopoulos N, Polyzos D, Chapman L, Magos A. The miniresectoscope: a new instrument for office hysteroscopic surgery. Acta Obstet Gynecol Scand 2009;88(2):227-30.

(9) Penketh RJ, Bruen EM, White J, Griffiths AN, Patwardhan A, Lindsay P, et al. Feasibility of resectoscopic operative hysteroscopy in a UK outpatient clinic using local anesthetic and traditional reusable equipment, with patient experiences and comparative cost analysis. J Minim Invasive Gynecol 2014 Sep;21(5):830-6.

(10) Emanuel MH, Wamsteker K. The Intra Uterine Morcellator: a new hysteroscopic operating technique to remove intrauterine polyps and myomas. J Minim Invasive Gynecol 2005 Jan;12(1):62-6.

(11) Munro MG. Hysteroscopic Myomectomy of FIGO Type 2 Leiomyomas Under Local Anesthesia: Bipolar Radiofrequency Needle-Based Release Followed By Electromechanical Morcellation. J Minim Invasive Gynecol 2016 Jan 1;23(1):12-3.

(12) Haimovich S, Mancebo G, Alameda F, Agramunt S, Sole-Sedeno JM, Hernandez JL, et al. Feasibility of a new two-step procedure for office hysteroscopic resection of submucous myomas: results of a pilot study. Eur J Obstet Gynecol Reprod Biol 2013 Jun;168(2):191-4.

(13) Bettocchi S, Di Spiezio SA, Ceci O, Nappi L, Guida M, Greco E, et al. A new hysteroscopic technique for the preparation of partially intramural myomas in office setting (OPPluM technique): A pilot study. J Minim Invasive Gynecol 2009 Nov;16(6):748-54.

(14) Haimovich S, Lopez-Yarto M, Urresta AJ, Saavedra TA, Hernandez JL, Carreras CR. Office Hysteroscopic Laser Enucleation of Submucous Myomas without Mass Extraction: A Case Series Study. Biomed Res Int 2015;2015:905204. 